\title{
Copula Theory: an Introduction
}

Fabrizio Durante and Carlo Sempi

\begin{abstract}
In this survey we review the most important properties of copulas, several families of copulas that have appeared in the literature, and which have been applied in various fields, and several methods of constructing multivariate copulas.
\end{abstract}

This version: September 14, 2009

\section{Historical introduction}

The history of copulas may be said to begin with Fréchet [69]. He studied the following problem, which is stated here in dimension 2: given the distribution functions $F_{1}$ and $F_{2}$ of two random variables $X_{1}$ and $X_{2}$ defined on the same probability space $(\Omega, \mathcal{F}, \mathbb{P})$, what can be said about the set $\Gamma\left(F_{1}, F_{2}\right)$ of the bivariate d.f.'s whose marginals are $F_{1}$ and $F_{2}$ ? It is immediate to note that the set $\Gamma\left(F_{1}, F_{2}\right)$, now called the Fréchet class of $F_{1}$ and $F_{2}$, is not empty since, if $X_{1}$ and $X_{2}$ are independent, then the distribution function $\left(x_{1}, x_{2}\right) \mapsto F\left(x_{1}, x_{2}\right)=F_{1}\left(x_{1}\right) F_{2}\left(x_{2}\right)$ always belongs to $\Gamma\left(F_{1}, F_{2}\right)$. But, it was not clear which the other elements of $\Gamma\left(F_{1}, F_{2}\right)$ were.

Preliminary studies about this problem were conducted in $[64,70,88]$ (see also $[30,181]$ for a historical overview). But, in 1959, Sklar obtained the deepest result in this respect, by introducing the notion, and the name, of a copula, and proving the theorem that now bears his name [191]. In his own words [193]:

Fabrizio Durante

Department of Knowledge-Based Mathematical Systems, Johannes Kepler University Linz, Austria e-mail: fabrizio.durante@jku.at

Carlo Sempi

Dipartimento di Matematica "Ennio De Giorgi"

Università del Salento, Lecce, Italy e-mail: carlo.sempi@unisalento.it 
[...] In the meantime, Bert (Schweizer) and I had been making progress in our work on statistical metric spaces, to the extent that Menger suggested it would be worthwhile for us to communicate our results to Fréchet. We did: Fréchet was interested, and asked us to write an announcement for the Comptes Rendus [183]. This began an exchange of letters with Fréchet, in the course of which he sent me several packets of reprints, mainly dealing with the work he and his colleagues were doing on distributions with given marginals. These reprints, among the later arrivals of which I particularly single out that of Dall'Aglio [28], were important for much of our subsequent work. At the time, though, the most significant reprint for me was that of Féron [64].

Féron, in studying three-dimensional distributions had introduced auxiliary functions, defined on the unit cube, that connected such distributions with their one-dimensional margins. I saw that similar functions could be defined on the unit $n$-cube for all $n \geq 2$ and would similarly serve to link $n$-dimensional distributions to their one-dimensional margins. Having worked out the basic properties of these functions, I wrote about them to Fréchet, in English. He asked me to write a note about them in French. While writing this, I decided I needed a name for these functions. Knowing the word "copula" as a grammatical term for a word or expression that links a subject and predicate, I felt that this would make an appropriate name for a function that links a multidimensional distribution to its one-dimensional margins, and used it as such. Fréchet received my note, corrected one mathematical statement, made some minor corrections to my French, and had the note published by the Statistical Institute of the University of Paris as Sklar [191].

The proof of Sklar's theorem was not given in [191], but a sketch of it was provided in [192] (see also [184]), so that for a few years practitioners in the field had to reconstruct it relying on the hand-written notes by Sklar himself; this was the case, for instance, of the second author. It should be also mentioned that some "indirect" proofs of Sklar's theorem (without mentioning copula) were later discovered by Moore and Spruill [144] and Deheuvels [36]

For about 15 years, all the results concerning copulas were obtained in the framework of the theory of Probabilistic Metric spaces [185]. The event that arose the interest of the statistical community in copulas occurred in the mid seventies, when Bert Schweizer, in his own words (see [182]),

quite by accident, reread a paper by A. Rényi, entitled On measures of dependence and realized that [he] could easily construct such measures by using copulas.

See [165] for Rényi's paper. The first building blocks were the announcement by Schweizer \& Wolff in the Comptes Rendus de l'Académie des Sciences [186] and Wolff's Ph.D. Dissertation at the University of Massachusetts at Amherst [199]. These results were presented to the statistical community in the paper [187] (compare also with [200]).

However, for several other years, Chapter 6 of the fundamental book [185] by Schweizer \& Sklar, devoted to the theory of Probabilistic metric spaces and published in 1983, was the main source of basic information on copulas. Again in Schweizer's words from [182],

After the publication of these articles and of the book ...the pace quickened as more ... students and colleagues became involved. Moreover, since interest in questions of statistical dependence was increasing, others came to the subject from different directions. In 1986 the enticingly entitled article The joy of copulas by C. Genest and R.C MacKay [81], attracted more attention. 
In 1990, Dall'Aglio organized the first conference devoted to copulas, aptly called "Probability distributions with given marginals" [31]. This turned out to be the first in a series of conferences that greatly helped the development of the field, since each of them offered the chance of presenting one's results and to learn those of other researchers; these conferences were held in Seattle in 1993 [175], in Prague in 1996 [11], in Barcelona in 2000 [25], in Québec in 2004 [74, 75], and in Tartu in 2007 [118]; the next one is scheduled to be in São Paulo in 2010.

At end of the nineties, the notion of copulas became increasingly popular. Two books about copulas appeared and were to become the standard references for the following decade. In 1997 Joe published his book on multivariate models [103], with a great part devoted to copulas and families of copulas. In 1999 Nelsen published the first edition of his introduction to copulas [149] (reprinted with some new results in [150]).

But, the main reason of this increased interest has to be found in the discovery of the notion of copulas by researchers in several applied field, like finance. Here we should like briefly to describe this explosion by quoting Embrechts's comments [56]:

As we have seen so far, the notion of copula is both natural as well as easy for looking at multivariate d.f.'s. But why do we witness such an incredible growth in papers published starting the end of the nineties (recall, the concept goes back to the fifties and even earlier, but not under that name). Here I can give three reasons: finance, finance, finance. In the eighties and nineties we experienced an explosive development of quantitative risk management methodology within finance and insurance, a lot of which was driven by either new regulatory guidelines or the development of new products; see for instance Chapter 1 in [137] for the full story on the former. Two papers more than any others "put the fire to the fuse": the [...] 1998 RiskLab report [57] and at around the same time, the Li credit portfolio model [120].

The advent of copulas in finance [78] originated a wealth of investigations about copulas and, especially, applications of copulas. See, for example, the books [180, $19,137,129]$. At the same time, different fields like hydrology [76, 176] discovered the importance of this concept for constructing more flexible multivariate models. Nowadays, it is near to impossible to give a complete account of all the applications of copulas to the many fields where they have be used. As Schweizer wrote [182]:

The "era of i.i.d." is over: and when dependence is taken seriously, copulas naturally come into play. It remains for the statistical community at large to recognize this fact. And when every statistics text contains a section or chapter on copulas, the subject will have come of age.

However, a word of caution is in order here. Several criticisms have been recently raised about copulas and their applications, and several people started to speak about "copula craze" [56]. See, for example, the very interesting discussion related to the paper by Mikosch [139, 140] (see also [55, 84, 92, 104, 125, 160, 188]).

From our point of view, these criticisms were a quite natural reaction to such a wide diffusion of applications of copulas, not always in a well motivated way. It should be said that several people have wrongly interpreted copulas as the solution 
to "all problems of stochastic dependence". This is definitely not the case! Copulas are an indispensable tool for understanding several problems about stochastic dependence, but they are not the "panacea" for all stochastic models.

Despite this broad range of interest about copulas, we still believe that this concept is still in "its infancy" [150] and several other investigations may (and should) be conducted in order to stress whether copulas, or related copula-based concepts, can be really considered as a "strong" mathematical concept worth of use in several applications (see Section 8).

\subsection{Outline of the paper}

This paper is so organized. Section 2 presents some basic notions of probability theory that will be used in the sequel. A basic introduction to copulas is given in section 3, while the importance of copulas for stochastic models is illustrated in sections 4 and 5. Families of copulas and construction methods are illustrated in section 6. Finally (section 8), we present a discussion about possible open problems in the field.

A final remark should be given here. This survey is intended to be a basic introduction to multivariate copulas, focusing on some theoretical aspects that we consider essential for understanding any application. We do not present any result about the statistical procedure for fitting copulas to data. The reader will find such an information in the rest of this book (see [12, 100, 179]). In writing it, we have tried to provide a list of references as complete as possible. Obviously, it may happen that several important papers have been not cited: we apologize in advance for this.

Other surveys concerning copulas (from various perspectives) can be found as well in the literature; just to make few same examples, we refer the interested reader to $[30,56,73,76,93,117,158,181,189,193,195]$.

\section{Preliminaries on random variables and distribution functions}

In this section, we recall the bare minimum that is necessary in order to understand the meaning and the use of copula. All this material can be found in standard books on probability theory, like [13, 105, 198].

To begin with, we need to establish basic notation. Let $d \in \mathbb{N}$. In the sequel, $\mathbf{x}$ denotes a vector $\left(x_{1}, x_{2}, \ldots, x_{d}\right)$ in $\mathbb{R}^{d}$ (or $\overline{\mathbb{R}}^{d}=[-\infty,+\infty]^{d}$ ). If not otherwise stated, all expressions such as $\min \{\mathbf{x}, \mathbf{y}\}$ or $\mathbf{x} \leq \mathbf{y}$ are intended to be componentwise operations. The symbol $\mathbb{I}$ will denote the unit interval $[0,1]$. We shall always use the expressions, "increasing" and "decreasing"in the weak sense; thus, a real function $\varphi$ defined on a subset $(a, b)$ of the real line $\mathbb{R}$, will be said to be increasing (respectively, decreasing) if, for all $x$ and $y$ in $(a, b)$ with $x<y$, one has $\varphi(x) \leq \varphi(y)$ 
(respectively, $\varphi(x) \geq \varphi(y)$ ). Moreover, a real-valued function $\varphi$ is said to be positive (respectively, strictly positive) if $\varphi(x) \geq 0$ (respectively, $\varphi(x)>0$ ) for every $x$ belonging to the domain of $\varphi$.

A probability space is a triplet $(\Omega, \mathcal{F}, \mathbb{P})$, where $\Omega$ is a nonempty set, $\mathcal{F}$ is a $\sigma$-algebra of subsets of $\Omega$ and $\mathbb{P}$ is a probability measure on $\mathcal{F}$. A $d$-dimensional random vector is a measurable mapping $\mathbf{X}: \Omega \rightarrow \overline{\mathbb{R}}^{d}$; in this case, the word measurable means that the counter image $\mathbf{X}^{-1}(B)$ of every Borel set $B$ in $\mathcal{B}\left(\overline{\mathbb{R}}^{d}\right)$ belongs to $\mathcal{F}$. It can be proved that a random vector $\mathbf{X}$ can be represented in the form $\mathbf{X}=\left(X_{1}, X_{2}, \ldots, X_{d}\right)$ where, for every $j \in\{1,2 \ldots, d\}, X_{j}$ is a 1 -dimensional random vector, also called random variable. Usually, the abbreviation r.v. will denote a random vector (possibly, univariate).

When a r.v. $\mathbf{X}=\left(X_{1}, X_{2}, \ldots, X_{d}\right)$ is given, two problems are interesting:

- to study the probabilistic behaviour of each one of its components;

- to investigate the relationship among them.

It will be seen how copulas allow to answer the second one of these problems in an admirable and thorough way.

It is a general fact that in probability theory, theorems are proved in the probability space $(\Omega, \mathcal{F}, \mathbb{P})$, while computations are usually carried out in the measurable space $\left(\overline{\mathbb{R}}^{d}, \mathcal{B}\left(\overline{\mathbb{R}}^{d}\right)\right)$ endowed with the law of the random vector $\mathbf{X}$. The study of the law $\mathbb{P}_{\mathbf{X}}$ is made easier by the knowledge of the distribution function, as defined here.

Definition 2.1. Given a random vector $\mathbf{X}=\left(X_{1}, X_{2}, \ldots, X_{d}\right)$ on the probability space $(\Omega, \mathcal{F}, \mathbb{P})$, its distribution function $F_{\mathbf{X}}: \overline{\mathbb{R}}^{d} \rightarrow \mathbb{I}$ is defined by

$$
F_{\mathbf{X}}\left(x_{1}, x_{2}, \ldots, x_{d}\right)=\mathbb{P}\left(\bigcap_{i=1}^{d}\left\{X_{i} \leq x_{i}\right\}\right)
$$

if all the $x_{i}$ 's are in $\mathbb{R}$, while:

(DF1) $\quad F_{\mathbf{X}}\left(x_{1}, x_{2}, \ldots, x_{d}\right)=0$, if at least one of the arguments equals $-\infty$,

(DF2) $\quad F_{\mathbf{X}}(+\infty,+\infty, \ldots,+\infty)=1$.

Very often, the abbreviation d.f. will be used instead of "distribution function". Sometimes, we also use the term joint d.f. for denoting the d.f. of a random vector having, at least, two components.

In order to describe the properties of a d.f., we need to introduce some preliminary notations.

Definition 2.2. Given two points $\mathbf{a}, \mathbf{b} \in \overline{\mathbb{R}}^{d}$ with $\mathbf{a} \leq \mathbf{b}$, a $d$-box $[\mathbf{a}, \mathbf{b}]$ (also called orthotope) is the cartesian product

$$
[\mathbf{a}, \mathbf{b}]=\stackrel{d}{\underset{i=1}{X}}\left[a_{i}, b_{i}\right]
$$


Let $A$ be a convex set in $\overline{\mathbb{R}}^{d}$. For a bounded function $H: A \rightarrow \overline{\mathbb{R}}$, the $H$-volume $V_{H}$ of the $d$-box $[\mathbf{a}, \mathbf{b}] \subseteq A$ is defined by

$$
V_{H}([\mathbf{a}, \mathbf{b}]):=\sum_{\mathbf{v} \in \chi_{i=1}^{d}\left\{a_{i}, b_{i}\right\}}(-1)^{N(\mathbf{v})} H(\mathbf{v}),
$$

where $N(\mathbf{v})=\operatorname{card}\left\{j \mid v_{i}=a_{i}\right\}$, provided that $a_{i}<b_{i}$ for every $i \in\{1,2, \ldots, d\}$. Otherwise, we set $V_{H}([\mathbf{a}, \mathbf{b}])=0$.

Theorem 2.1. The d.f. $F_{\mathbf{X}}$ of the r.v. $\mathbf{X}=\left(X_{1}, X_{2}, \ldots, X_{d}\right)$ has the following properties:

(DF3) $\quad F$ is isotonic, i.e. $F(\mathbf{x}) \leq F(\mathbf{y})$ for all $\mathbf{x}, \mathbf{y} \in \mathbb{R}^{d}, \mathbf{x} \leq \mathbf{y}$;

(DF4) for all $\left(x_{1}, \ldots, x_{i-1}, x_{i+1}, \ldots, x_{d}\right) \in \mathbb{R}^{d-1}$, the function

$$
\mathbb{R} \ni t \mapsto F_{X}\left(x_{1}, \ldots, x_{i-1}, t, x_{i+1}, \ldots, x_{d}\right)
$$

is right-continuous;

(DF5) the $F_{\mathbf{X}}$-volume $V_{F_{\mathbf{X}}}$ of every $d-$ box $[\mathbf{a}, \mathbf{b}]$ is positive, i.e., $V_{F_{\mathbf{X}}}([\mathbf{a}, \mathbf{b}]) \geq 0$.

Condition (DF5) in the previous theorem is called the $d$-increasing property (or quasi-monotone property) of a $d$-dimensional d.f.; it simply means that the probability that the r.v. $\mathbf{X}$ takes values in the box $[\mathbf{a}, \mathbf{b}]$ is positive. Analogously, a function $F: \overline{\mathbb{R}}^{d} \rightarrow \mathbb{R}$ satisfies (DF5) if, and only if, it has positive finite differences of order $d$, namely if

$$
\Delta_{a_{d}, b_{d}}^{d} \ldots \Delta_{a_{1}, b_{1}}^{1} F \geq 0
$$

for all $a_{i} \leq b_{i}$ for every $i \in\{1,2, \ldots, d\}$, where $\Delta_{a_{i}, b_{i}}^{i}$ is the finite difference operator given by

$$
\Delta_{a_{i}, b_{i}}^{i} F=F\left(t_{1}, \ldots, t_{i-1}, b_{i}, t_{i+1}, \ldots, t_{d}\right)-F\left(t_{1}, \ldots, t_{i-1}, a_{i}, t_{i+1}, \ldots, t_{d}\right) .
$$

Note that, one can prove (see, e.g., [13]) that for every $F: \mathbb{R}^{d} \rightarrow \mathbb{I}$ satisfying conditions (DF1)-(DF5), there is a probability space $(\Omega, \mathcal{F}, \mathbb{P})$ and a random vector $\mathbf{X}$ on it such that $F$ is the d.f. of $\mathbf{X}$. We will use the symbol $\mathbf{X} \sim F$ in order to denote the fact that $F$ is the d.f. of $\mathbf{X}$ (or, equivalently, $\mathbf{X}$ is distributed according to $F$ ).

A fundamental notion will be that of marginal distribution of a $d$-d.f. $F$.

Definition 2.3. Let $d \geq 2$ and let $F$ be a $d$-dimensional d.f.. Let $\sigma=\left(j_{1}, \ldots, j_{m}\right)$ a subvector of $(1,2, \ldots, d), 1 \leq m \leq d-1$. We call $\sigma$-marginal of $F$ the d.f. $F_{\sigma}: \overline{\mathbb{R}}^{m} \rightarrow \mathbb{I}$ defined by setting $d-m$ arguments of $F$ equal to $+\infty$, namely, for every $x_{1}, \ldots, x_{m} \in$ $\overline{\mathbb{R}}$,

$$
F_{\sigma}\left(x_{1}, \ldots, x_{m}\right)=F\left(y_{1}, \ldots, y_{d}\right),
$$

where, for every $j \in\{1,2, \ldots, d\}, y_{j}=x_{j}$ if $j \in\left\{j_{1}, \ldots, j_{m}\right\}$, and $y_{j}=+\infty$ otherwise. 
In particular, when $\sigma=\{j\}, F_{(j)}$ is usually called 1-dimensional marginal and it is denoted by $F_{j}$.

Obviously, if $F$ is the d.f. of the r.v. $\mathbf{X}=\left(X_{1}, X_{2}, \ldots, X_{d}\right)$, then the $\sigma$-marginal of $F$ is simply the d.f. of the subvector $\left(X_{j_{1}}, \ldots, X_{j_{m}}\right)$.

As is well known, if the r.v.'s $X_{1}, X_{2}, \ldots, X_{d}$ are independent and if $F_{i}$ denotes the d.f. of $X_{i}(i=1,2, \ldots, d)$, then the $d$-dimensional d.f. of the random vector $\mathbf{X}=$ $\left(X_{1}, X_{2}, \ldots, X_{d}\right)$ is the product of the marginals, i.e. for all $x_{1}, x_{2}, \ldots, x_{d} \in \overline{\mathbb{R}}$,

$$
F_{\mathbf{X}}\left(x_{1}, x_{2}, \ldots, x_{d}\right)=\prod_{i=1}^{d} F_{i}\left(x_{j}\right)
$$

A random vector $\mathbf{X}$ (equivalently, its d.f. $F_{\mathbf{X}}$ ) is said to be absolutely continuous if there exists a positive measurable function $f_{\mathbf{X}}: \mathbb{R}^{d} \rightarrow \mathbb{R}_{+}$(called the density of $F$ ) such that

$$
\int_{\mathbb{R}^{d}} f_{\mathbf{X}} d \lambda_{d}=1
$$

where $\lambda_{d}$ is the Lebesgue measure on $\mathbb{R}^{d}$, and the d.f. $F_{\mathbf{X}}$ can be represented in the form

$$
F_{\mathbf{X}}(\mathbf{x})=\int_{-\infty}^{x_{1}} d t_{1} \int_{-\infty}^{x_{2}} d t_{2} \ldots \int_{-\infty}^{x_{d}} f_{X}\left(t_{1}, t_{2}, \ldots, t_{d}\right) d t_{d} .
$$

For a univariate absolutely continuous d.f. $F$, we shall use the notion $F \sim \mathcal{U}([a, b])$ (read: $F$ is uniformly distributed on $[a, b]$ ) to denote the fact that $F$ is absolutely continuous with density $f(t)=(b-a)^{-1} \mathbf{1}_{(a, b)}(t)$, where $\mathbf{1}_{(a, b)}$ denoted the indicator function of $(a, b)$.

Remark 2.1. Let $\mathbf{X}$ be a r.v. such that $\mathbb{P}(\mathbf{X} \in A)=1$ for some $A \subseteq \mathbb{R}^{d}$. Then, the d.f. $F_{\mathbf{X}}$ is uniquely determined by the value that it assumes on $A$ and, as a consequence, one usually refrains from specifying the value of $F_{\mathbf{X}}$ outside $A$. For such a situation, we may say shortly that $F_{\mathbf{X}}$ is a d.f. on $A$.

Remark 2.2. In several contexts (especially, reliability theory), it is more useful to consider the survival function $\bar{F}_{\mathbf{X}}$ associated with a given random vector $\mathbf{X}=$ $\left(X_{1}, X_{2}, \ldots, X_{d}\right)$, where, for every $i \in\{1,2, \ldots, d\}, X_{i} \geq 0$ almost surely (i.e., $X_{i}$ can be interpreted as a "lifetime"), and given by

$$
\bar{F}_{\mathbf{X}}\left(x_{1}, x_{2}, \ldots, x_{d}\right)=\mathbb{P}\left(\bigcap_{i=1}^{d}\left\{X_{i}>x_{i}\right\}\right) .
$$

The univariate survival marginal of $\bar{F}_{\mathbf{X}}$ are then defined in an analogous way by setting some of the arguments of $\bar{F}$ equal to 0 . Note that survival functions are decreasing in each argument. 


\section{Copulas: definitions and basic properties}

We start with the definition of a copula.

Definition 3.1. For every $d \geq 2$, a $d$-dimensional copula (shortly, $d$-copula) is a $d$ variate d.f. on $\mathbb{I}^{d}$ whose univariate marginals are uniformly distributed on $\mathbb{I}$.

Thus, each $d$-copula may be associated with a r.v. $\mathbf{U}=\left(U_{1}, U_{2}, \ldots, U_{d}\right)$ such that $U_{i} \sim \mathcal{U}(\mathbb{I})$ for every $i \in\{1,2, \ldots, d\}$ and $\mathbf{U} \sim C$. Conversely, any r.v. whose components are uniformly distributed on $\mathbb{I}$ is distributed according to some copula.

The class of all $d$-copulas will be denoted by $\mathcal{C}_{d}$.

Since copulas are multivariate d.f.'s, as a consequence of the results stated in section 2, they can be characterized in the following equivalent way.

Theorem 3.1. A function $C: \mathbb{I}^{d} \rightarrow \mathbb{I}$ is a copula if, and only if, the following properties hold:

(C1) for every $j \in\{1,2, \ldots, d\}, C(\mathbf{u})=u_{j}$ when all the components of $\mathbf{u}$ are equal to 1 with the exception of the $j$-th one that is equal to $u_{j} \in \mathbb{I}$;

(C2) $\quad C$ is isotonic, i.e. $C(\mathbf{u}) \leq C(\mathbf{v})$ for all $\mathbf{u}, \mathbf{v} \in \mathbb{I}^{d}, \mathbf{u} \leq \mathbf{v}$;

(C3) $\quad C$ is $d$-increasing.

As an easy consequence, we can prove also that $C(\mathbf{u})=0$ for every $\mathbf{u} \in \mathbb{I}^{d}$ having at least one of its components equal to 0 . Another interesting property of a $d$-copula $C$ is that it is a Lipschitz function, namely, for all $\mathbf{u}, \mathbf{v} \in \mathbb{I}^{d}$, one has

$$
|C(\mathbf{u})-C(\mathbf{v})| \leq \sum_{i=1}^{d}\left|u_{i}-v_{i}\right| .
$$

By using Ascoli-Arzelá Theorem, one can show that $\mathcal{C}_{d}$ is a compact set in the set of all continuous functions from $\mathbb{I}^{d}$ into $\mathbb{I}$ equipped with the product topology, which corresponds to the topology of pointwise convergence. Moreover, in $\mathcal{C}_{d}$ pointwise and uniform convergence are equivalent (see also [32]).

Basic examples of copulas are:

- the independence copula $\Pi_{d}(\mathbf{u})=u_{1} u_{2} \cdots u_{d}$ associated with a random vector $\mathbf{U}=$ $\left(U_{1}, U_{2}, \ldots, U_{d}\right)$ whose components are independent and uniformly distributed on I;

- the comonotonicity copula $M_{d}(\mathbf{u})=\min \left\{u_{1}, u_{2}, \ldots, u_{d}\right\}$ associated with a vector $\mathbf{U}=\left(U_{1}, U_{2}, \ldots, U_{d}\right)$ of r.v.'s uniformly distributed on $\mathbb{I}$ and such that $U_{1}=U_{2}=$ $\cdots=U_{d}$ almost surely;

- the countermonotonicity copula $W_{2}\left(u_{1}, u_{2}\right)=\max \left\{u_{1}+u_{2}-1,0\right\}$ associated with a vector $\mathbf{U}=\left(U_{1}, U_{2}\right)$ of r.v.'s uniformly distributed on $\mathbb{I}$ and such that $U_{1}=1-U_{2}$ almost surely.

By using Theorem 3.1 it is easy to show that the set $\mathcal{C}_{d}$ is convex. Convex combinations of copulas have the following probabilistic interpretation (compare with [142]). 
Example 3.1 (Convex combinations of copulas). Let $\mathbf{U}^{1}$ and $\mathbf{U}^{2}$ be two $d$-dimensional r.v.'s on the probability space $(\Omega, \mathcal{F}, \mathbb{P})$ distributed according to the copulas $C^{1}$ and $C^{2}$, respectively. Let $Z$ be a Bernoulli r.v. such that $\mathbb{P}(Z=1)=\alpha$ and $\mathbb{P}(Z=$ $2)=1-\alpha$ for some $\alpha \in \mathbb{I}$. Suppose that $\mathbf{U}^{1}, \mathbf{U}^{2}$ and $Z$ are independent. Now, consider the $d$-dimensional r.v. $\mathbf{U}^{*}$

$$
\mathbf{U}^{*}=\sigma_{1}(Z) \mathbf{U}^{1}+\sigma_{2}(Z) \mathbf{U}^{2}
$$

where, for $i \in\{1,2\}, \sigma_{i}(x)=1$, if $x=i, \sigma_{i}(x)=0$, otherwise. Then, it could be proved that $\mathbf{U}^{*}$ is distributed according to the copula $\alpha C^{1}+(1-\alpha) C^{2}$.

Example 3.2 (Fréchet-Mardia copulas). Let $C_{d}^{\mathrm{FM}}$ be the $d$-copula given by

$$
C_{d}^{\mathbf{F M}}(\mathbf{u})=\alpha \Pi_{d}(\mathbf{u})+(1-\alpha) M_{d}(\mathbf{u})
$$

for every $\lambda \in \mathbb{I}$. These copulas can be considered as a multivariate version of the bivariate families by Fréchet and Mardia (see [71, 131]). They are obtained as convex sum of the copulas $\Pi_{d}$ and $M_{d}$.

Example 3.3. Let $\mathbf{X}=\left(X_{1}, X_{2}, \ldots, X_{d}\right)$ be a r.v. whose components are independent and identically distributed according to $F_{X_{i}}(t)=t^{\alpha}$ for every $t \in \mathbb{I}$ and for some $\alpha \in \mathbb{I}$. Let $Z$ be a random variable, independent of $\mathbf{X}$, whose d.f. is given by $F_{Z}(t)=t^{1-\alpha}$ on $\mathbb{I}$. Intuitivelly, $Z$ might be interpreted as a shock that will change the dependence structure of $\mathbf{X}$. We define another r.v. $\mathbf{Y}$ such that, for every $i \in\{1,2, \ldots, d\}, Y_{i}=$ $\max \left\{X_{i}, Z\right\}$. Now, it can be easily proved that the joint d.f. of $Y$ is, in fact, a copula given by

$$
C_{d}^{\mathbf{C A}} \mathbf{u}=\left(\Pi_{d}(\mathbf{u})\right)^{\alpha}\left(M_{d}(\mathbf{u})\right)^{1-\alpha},
$$

which belongs to the Cuadras-Augé family of copulas (see [24] and also [23]). The idea of considering families of d.f.'s associated with some shock model had its origin in the seminal paper by Marshall and Olkin (see [133]), where the multivariate exponential distribution was considered (see also [121, 122, 147])). Further generalizations of these methods have recently provided several constructions of copulas, as can be seen from $[43,49,126,127,128]$.

The following result gives upper and lower bounds in $\mathcal{C}_{d}$ (see, for example, [150, Theorem 2.10.12]).

Theorem 3.2 (Fréchet-Hoeffding bounds). For every $C_{d} \in \mathcal{C}_{d}$ and for every $\mathbf{u} \in \mathbb{I}^{d}$,

$$
W_{d}(\mathbf{u})=\max \left\{\sum_{i=1}^{d} u_{i}-d+1,0\right\} \leq C(\mathbf{u}) \leq M_{d}(\mathbf{u}) .
$$

Moreover, the following bounds are sharp, in the sense that the pointwise infimum and supremum of all the elements of $\mathcal{C}_{d}$ coincide, respectively, with $W_{d}$ and $M_{d}$, i.e. for all $\mathbf{u} \in \mathbb{I}^{d}$ :

$$
\inf _{C \in \mathcal{C}_{d}} C(\mathbf{u})=W_{d}(\mathbf{u}), \quad \sup _{C \in \mathcal{C}_{d}} C(\mathbf{u})=M_{d}(\mathbf{u}) .
$$


Notice that, while $W_{2}$ is a copula, $W_{d}$ is not a copula for $d \geq 3$.

The Fréchet-Hoeffding bounds appeared for the first time in the present form in an article by Fréchet (see [69]). An earlier version had already be given by Hoeffding ([94]), but with reference to the square $[-1 / 2,1 / 2]^{2}$. A very general formulation of it, including the proof of the sharpness of these bounds, has been given by Rüschendorf [172].

Several investigations have been conducted about the bounds for multivariate d.f.'s (see [15, 153, 168]), especially when some additional information is given, like lower dimensional marginals [37, 45, 103], and measures of association [151, 155]. In this context, the concept of quasi-copula plays a special rôle $[2,26,83,152,156$, 171].

\section{Sklar's Theorem}

Sklar's theorem is the building block of the theory of copulas; without it, the concept of copula would be one in a rich set of joint distribution functions.

Theorem 4.1. Let $F$ be a d-dimensional d.f. with univariate margins $F_{1}, F_{2}, \ldots, F_{d}$. Let $A_{j}$ denote the range of $F_{j}, A_{j}:=F_{j}(\overline{\mathbb{R}})(j=1,2, \ldots, d)$. Then there exists a copula $C$ such that for all $\left(x_{1}, x_{2}, \ldots, x_{d}\right) \in \overline{\mathbb{R}}^{d}$,

$$
F\left(x_{1}, x_{2}, \ldots, x_{d}\right)=C\left(F_{1}\left(x_{1}\right), F_{2}\left(x_{2}\right), \ldots, F_{d}\left(x_{d}\right)\right) .
$$

Such a $C$ is uniquely determined on $A_{1} \times A_{2} \times \cdots \times A_{d}$ and, hence, it is unique when $F_{1}, F_{2}, \ldots, F_{d}$ are all continuous.

Sklar's theorem has been announced in [191], however its first proof for the bivariate case appeared in [184]. Curiously, it should be noted that in [191], the author "Abe Sklar" is named as "M. Sklar" (we conjecture that this "M." should be intended as "Monsieur").

Another (bivariate) proof can be also found in [16], based on the so-called "checkerboard copulas". A multivariate proof, based on the distributional transform, has been recently presented in [174] (compare also with [144, Lemma 3.2]). Another possible proof can be also derived (for positive random variables) from [14]. Sklar's Theorem on more abstract spaces has been given in [178].

Theorem 4.1 also admits the following converse implication, usually very important when one wants to construct statistical models by considering, separately, the univariate behaviour of the components of a random vector and their dependence properties as captured by some copula.

Theorem 4.2. If $F_{1}, F_{2}, \ldots, F_{d}$ are univariate d.f.'s, and if $C$ is any $d$-copula, then the function $F: \overline{\mathbb{R}}^{d} \rightarrow \mathbb{I}$ defined by (11) is a d-dimensional distribution function with margins $F_{1}, F_{2}, \ldots, F_{d}$. 
By summarizing, from any $d$-variate d.f. $F$ one can derive a copula $C$ via (11). Specifically, when $F_{i}$ is continuous for every $i \in\{1,2, \ldots, d\}, C$ can be obtained by means of the formula

$$
C\left(u_{1}, u_{2}, \ldots, u_{d}\right)=F\left(F_{1}^{-1}\left(u_{1}\right), F_{2}^{-1}\left(u_{2}\right), \ldots, F_{d}^{-1}\left(u_{d}\right)\right),
$$

where $F_{i}^{-1}$ denoted the pseudo-inverse of $F_{i}$ given by $F_{i}^{-1}(s)=\inf \left\{t \mid F_{i}(t) \geq s\right\}$. Thus, copulas are essentially a way for transforming the r.v. $\left(X_{1}, X_{2} \ldots, X_{d}\right)$ into another r.v. $\left(U_{1}, U_{2}, \ldots, U_{d}\right)=\left(F_{1}\left(X_{1}\right), F_{2}\left(X_{2}\right), \ldots, F_{d}\left(X_{d}\right)\right)$ having the margins uniform on $\mathbb{I}$ and preserving the dependence among the components.

On the other hand, any copula can be combined with different univariate d.f.'s in order to obtain a $d$-variate d.f. by using (11). In particular, copulas can serve for modelling situations where a different distribution is needed for each marginal, providing a valid alternative to several classical multivariate d.f.'s such Gaussian, Pareto, Gamma, etc. (compare with [73]). This fact represents one of the main advantage of the copula's idea, as underlined by Mikosch [139]:

There is no simple alternative to the Gaussian distribution in the non-Gaussian world. In particular, one needs multivariate models for portfolios with different marginal distributions (including different tail behavior) and a dependence structure which is determined not only by covariances. Many of the well known multivariate distributions are not flexible enough to allow for different tail behavior in different components. Therefore copulas seem to be the right tools in order to overcome the mentioned difficulties: they generate all multivariate distributions with flexible marginals.

Remark 4.1. The copula representation is usually very convenient, as stressed for example by Kimeldorf and Sampson [108], who referred to it as uniform representation. However, following [56], it should be stressed that

there is absolutely no real, compelling mathematical reason for transforming the marginal d.f.'s of $F$ to uniform d.f.'s on $\mathbb{I}$, though it may be useful from a statistical point of view. In his 1940 paper [96], Hoeffding used the interval $\left[-\frac{1}{2}, \frac{1}{2}\right]$. In multivariate Extreme Value Theory, it is standard to transform to unit Fréchet marginal d.f.'s. In this context, Resnick [166, page 265] writes "How one standardizes is somewhat arbitrary and depends on taste. Different specifications have led to (superficially) different representations in the literature".

As investigated recently in [114], other ways for transforming r.v.'s to having some standard margins may be more convenient in some cases.

Remark 4.2. Theorem 4.1 should be used with some caution when the margins have jumps. In fact, even if there exists a copula representation for not-continuous joint d.f.'s, it is no longer unique. In such cases, modelling and interpreting dependence through copulas is subject to caution. The interested readers should refer to the seminal paper by Marshall [132] and to the in-depth discussion by Genest and Nešlehová [82].

Finally, notice that Theorems 4.1 and 4.2 can be formulated in an analogous way in terms of survival functions instead of d.f.'s. Specifically, given a r.v. $\mathbf{X}=$ $\left(X_{1}, X_{2}, \ldots X_{d}\right)$ with joint survival function $\bar{F}$ and univariate survival marginals $\bar{F}_{i}$ $(i=1,2, \ldots, d)$, for all $\left(x_{1}, x_{2}, \ldots, x_{n}\right) \in \overline{\mathbb{R}}^{d}$ it holds that: 


$$
\bar{F}\left(x_{1}, x_{2}, \ldots, x_{d}\right)=\widehat{C}\left(\bar{F}_{1}\left(x_{1}\right), \bar{F}_{2}\left(x_{2}\right), \ldots, \bar{F}_{d}\left(x_{d}\right)\right) .
$$

for some copula $\widehat{C}$, usually called survival copula of $\mathbf{X}$ (to be intended as a copula associated with the survival function of $\mathbf{X}$ ).

In particular, let $C$ be the copula of $\mathbf{X}$ and let $\mathbf{U}=\left(U_{1}, U_{2}, \ldots, U_{d}\right)$ be a vector such that $\mathbf{U} \sim C$. Then, one has

$$
\widehat{C}(\mathbf{u})=\bar{C}\left(1-u_{1}, 1-u_{2}, \ldots, 1-u_{d}\right),
$$

where $\bar{C}(\mathbf{u})=\mathbb{P}\left(U_{1}>u_{1}, U_{2}>u_{2}, \ldots, U_{d}>u_{d}\right)$ is the survival function associated with $C$, explicitly given by

$$
\bar{C}(\mathbf{u})=1+\sum_{k=1}^{d}(-1)^{k} \sum_{1 \leq i_{1}<i_{2}<\cdots<i_{k} \leq n} C_{i_{1} i_{2} \cdots i_{k}}\left(u_{i_{1}}, u_{i_{2}}, \ldots, u_{i_{k}}\right),
$$

with $C_{i_{1} i_{2} \cdots, i_{k}}$ denoting the marginal of $C$ related to $\left(i_{1}, i_{2}, \cdots, i_{k}\right)$.

\section{Copulas and random vectors}

Here, we should like to discuss some probabilistic properties of copulas that can be inferred from Sklar's Theorem. These results appeared already in [192] (for the proofs, see $[137,150])$.

First, we state the invariance of the copula of the r.v. $\mathbf{X}$ with respect to any increasing rescaling of the components of $\mathbf{X}$.

Proposition 5.1. Let $\mathbf{X}=\left(X_{1}, \ldots, X_{d}\right)$ be a r.v. with continuous d.f. $F$, univariate marginals $F_{1}, F_{2} \ldots, F_{d}$, and copula $C$. Let $T_{1}, \ldots, T_{d}$ be strictly increasing functions from $\mathbb{R}$ to $\mathbb{R}$. Then $C$ is also the copula of the r.v. $\left(T_{1}\left(X_{1}\right), \ldots, T_{d}\left(X_{d}\right)\right)$.

Thus, copulas that describe the dependence of the components of a random vector are invariant under increasing transformations of each coordinate. Therefore, as said in [187],

the study of rank statistics - insofar as it is the study of properties invariant under such transformations - may be characterized as the study of copulas and copula-invariant properties.

The next results characterize some special structures of r.v.'s in terms of the basic copulas $\Pi_{d}, M_{d}$, and $W_{2}$.

Proposition 5.2. Let $\left(X_{1}, X_{2}, \ldots, X_{d}\right)$ be a r.v. with continuous joint $d . f . F$ and univariate marginals $F_{1}, \ldots, F_{d}$. Then the copula of $\left(X_{1}, \ldots, X_{d}\right)$ is $\Pi_{d}$ if, and only if, $X_{1}, \ldots, X_{d}$ are independent.

Proposition 5.3. Let $\left(X_{1}, X_{2}, \ldots, X_{d}\right)$ be a r.v. with continuous joint d.f. $F$ and univariate marginals $F_{1}, \ldots, F_{d}$. Then the copula of $\left(X_{1}, \ldots, X_{d}\right)$ is $M_{d}$ if, and 
only if, there exists a r.v. $Z$ and increasing functions $T_{1}, \ldots, T_{d}$ such that $\mathbf{X}=$ $\left(T_{1}(Z), \ldots, T_{d}(Z)\right)$ almost surely.

Proposition 5.4. Let $\left(X_{1}, X_{2}\right)$ be a r.v. with continuous $d . f . F$ and univariate marginals $F_{1}, F_{2}$. Then $\left(X_{1}, X_{2}\right)$ has copula $W_{2}$ if, and only if, for some strictly decreasing function $T, X_{2}=T\left(X_{1}\right)$ almost surely.

Obviously Proposition 5.4 cannot be extended to dimension $d$ with $d \geq 3$; in fact, it is impossible for each one of the $d$ random variables $X_{1}, X_{2}, \ldots, X_{d}$ to be a decreasing function of the remaining ones.

A r.v. $\mathbf{X}$ as in Proposition 5.3 is said to have a comonotone dependence. This concept has several important consequences in applications, as described in $[38,39]$. The related concept described in Proposition 5.4 is known as countermonotonicity.

A recent generalization of comonotonicity has been provided in [161].

\section{Families of copulas}

As underlined, copulas play an important role in the construction of multivariate d.f.'s and, as a consequence, having at one's disposal a variety of copulas can be very useful for building stochastic models having different properties that are sometimes indispensable in practice (e.g., heavy tails, asymmetries, etc.). Therefore, several investigations have been carried out concerning the construction of different families of copulas and their properties. Here, we present just a few of them, by focusing on the families that seem to be more popular in the literature. Different families (or construction methods) are discussed in the rest of this book (see [27, 87, 95]).

Before presenting these examples, we would like briefly to discuss the use of these families for copula-fitting procedures. For several researchers and practitioners it seems enough to try to fit any stochastic model with the most convenient family and check that the fitting procedure is not so "bad". However, it should be stressed that any fitting procedure may be misleading if one were to describe any situation with families of copulas satisfying some unnecessary assumptions (e.g., exchangeability, light tails, etc.). In our opinion, before applying any statistical tool, one should not forget to analyse the main characteristics of the model under consideration. At the same time, one should have clearly in mind the final output of the investigation. Usually, in fact, fitting a copula (or a joint d.f.) to some data is just a tool for deriving some quantities of interest for the problem at hand (e.g., VaR of a portfolio, return period of an extreme event, etc.). For such problems, a dramatic underestimation of the risk can be obtained when one tries to fit with copulas that do not exhibit any peculiar behaviour in the tails. This was exactly one of the main criticisms to the use of Li's model for credit risk using Gaussian copulas (see, for example, $[120,197])$.

Inspired by some considerations of Joe [103], we discuss here some general properties that a "good" family of multivariate copulas $\left\{C_{\theta}\right\}$, where $\theta$ is a parameter 
belonging to a (usually, compact) subset $\Theta \subseteq \mathbb{R}^{p}(p \geq 1)$, should have for being considered "interesting" in statistical applications.

- Interpretability. The members of the family should have some probabilistic interpretation suggesting "natural" situations where this family could be considered. For instance, as we have seen in Example 3.3, Cuadras-Augé copulas have a quite direct interpretation in terms of shock models, making this class suitable for modelling situations where a common shock has consequences on a system composed by several components (credit portfolio, lifetime system, etc.).

- Flexible and wide range of dependence. The members of the family should describe different types of dependence, including the independence copula $\Pi_{d}$ and one of the Fréchet-Hoeffding bounds (possibly, as a limiting case with respect to the parameter). Having members with a variety of tail dependencies and asymmetries is a desirable property as well.

- Easy-to-handle. The members of the family should be expressed in a closed form or, at least, should be easily simulated by means of some known algorithm. Notice, in fact, that several goodness-of-fit procedures are based on the fact that the fitted family can be readily sampled (see [12] and the references therein).

Notice that several families of copulas recently introduced seem not to be "good" in the above sense. This should be not surprising. On one hand, the above properties are intentionally be given in a very restrictive way, on the other hand, one should consider that several new constructions have been introduced and studied more for their mathematical elegance and interest than for a practical implementation. It is our opinion that in the future more attention should be devoted to this latter aspect: there are no clever applications without a beautiful theory!

\subsection{Elliptical copulas}

A random vector $\mathbf{X}=\left(X_{1}, X_{2} \ldots, X_{d}\right)$ is said to have an elliptical distribution with mean vector $\mu \in \mathbb{R}^{d}$, covariance matrix $\Sigma=\left(\sigma_{i j}\right)$ and generator $g:[0,+\infty[\rightarrow[0,+\infty[$, and one writes $\mathbf{X} \sim \mathcal{E}(\mu, \Sigma, g)$, if it can be expressed in the form

$$
\mathbf{X}=\mu+R \mathbf{A U}
$$

where $\mathbf{A} \mathbf{A}^{\mathbf{T}}=\Sigma$ is the Cholesky decomposition of $\Sigma, \mathbf{U}$ is a $d$-dimensional random vector uniformly distributed on the sphere $\mathbb{S}^{d-1}=\left\{\mathbf{u} \in \mathbb{R}^{d}: u_{1}^{2}+\cdots+u_{d}^{2}=1\right\}$, and $R$ is a positive random variable independent of $\mathbf{U}$, with density given, for every $r>0$, by

$$
f_{g}(r)=\frac{2 \pi^{d / 2}}{\Gamma(d / 2)} r^{d-1} g\left(r^{2}\right) .
$$

The density function (if it exists) of an elliptical distribution is given, for every $\mathbf{x} \in \mathbb{R}^{d}$, by

$$
h_{g}(\mathbf{x})=|\Sigma|^{-1 / 2} g\left((\mathbf{x}-\mu)^{T} \Sigma^{-1}(\mathbf{x}-\mu)\right) \text {. }
$$


For more details, see [61, 62, 67, 77, 137].

For instance, when $g(t)=(2 \pi)^{-d / 2} \exp (-t / 2)$, then $\mathbf{X}$ has a multivariate Gaussian distribution. Similarly, $g(t)=c(1+t / v)^{-(d+v) / 2}$, for a suitable constant $c$, generates the multivariate $t$-Student distribution with $v$ degrees of freedom.

One of the characteristics of an elliptical distribution is that the scaled components $X_{1} / \sqrt{\sigma_{11}}, \ldots, X_{d} / \sqrt{\sigma_{d d}}$ are identically distributed according to a d.f. $F_{g}$. This fact represents a limitation to the use of such distributions for modelling stochastic systems when the components are not similar. In order to avoid this, it is useful to calculate the copula of a multivariate elliptical distributions and use it, together with some univariate marginal d.f.'s, for obtaining more flexible models. These distributions, constructed by means of Theorem 4.2, are usually called meta-elliptical distribution [62]. For these reasons, we give the following

Definition 6.1. Let $\mathbf{X}$ be an elliptical random vector, $\mathbf{X} \sim \mathcal{E}_{d}(\mu, \Sigma, g)$. Suppose that, for every $i \in\{1,2, \ldots, d\},\left(X_{i} / \sqrt{\sigma_{i i}}\right) \sim F_{g}$. We call elliptical copula the distribution function of the random vector

$$
\left(F_{g}\left(\frac{X_{1}}{\sqrt{\sigma_{11}}}\right), F_{g}\left(\frac{X_{2}}{\sqrt{\sigma_{22}}}\right), \ldots, F_{g}\left(\frac{X_{d}}{\sqrt{\sigma_{d d}}}\right)\right) .
$$

An elliptical copula is typically not available in closed form. In order just to provide an example, the bivariate Gaussian copula is given by

$$
C_{\theta}\left(u_{1}, u_{2}\right)=\int_{-\infty}^{\Phi^{-1}\left(u_{1}\right)} \int_{-\infty}^{\Phi^{-1}\left(u_{2}\right)} \frac{1}{2 \pi \sqrt{1-\theta^{2}}}\left(-\frac{s^{2}-2 \theta s t+s^{2}}{2\left(1-\theta^{2}\right)}\right) \mathrm{d} s \mathrm{~d} t
$$

where $\theta \in[-1,1]$, and $\Phi^{-1}$ denotes the inverse of the univariate Gaussian distribution.

\subsection{Archimedean copulas}

Here we present the basic properties and examples of the Archimedean class of copulas. Basically, we follow the approach in [136] (see also [135]).

First, we introduce some notations. We call Archimedean generator any decreasing and continuous function $\psi:[0, \infty[\rightarrow \mathbb{I}$ that satisfies the conditions $\psi(0)=1$, $\lim _{t \rightarrow \infty} \psi(t)=0$ and which is strictly decreasing on $[0, \inf \{t \mid \psi(t)=0\}[$. By convention, $\psi(+\infty)=0$ and $\psi^{-1}(0)=\inf \{t \geq 0 \mid \psi(t)=0\}$, where $\psi^{-1}$ denotes the pseudo-inverse of $\psi$.

Definition 6.2. A $d$-dimensional copula $C$ is called Archimedean if it admits the representation

$$
C(\mathbf{u})=\psi\left(\psi^{-1}\left(u_{1}\right)+\psi^{-1}\left(u_{2}\right)+\cdots+\psi^{-1}\left(u_{d}\right)\right)
$$

for all $\mathbf{u} \in \mathbb{I}^{d}$ and for some Archimedean generator $\psi$. 
Functions of type (17) have been widely considered and studied in the literature, especially in connexion with the investigations about semigroups of the unit interval. The reader interested about all these aspects should look at the following books $[1,111,185]$ and the references therein.

The following result characterizes Archimedean copulas in terms of the properties of their generators; it can be found in [136].

Theorem 6.1. Let $\psi$ be an Archimedean generator. Let $C_{\psi}$ be the function given by (17). Then $C_{\psi}$ is a d-dimensional copula if, and only if, the restriction of $\psi$ to ] $0, \infty[$ is $d$-monotone, i.e. it satisfies:

(a) $\psi$ is differentiable up to the order $d-2$ in $] 0,+\infty[$ and the derivatives satisfy

$$
(-1)^{k} \psi^{(k)}(t) \geq 0 \quad \text { for } k \in\{0,1, \ldots, d-2\}
$$

for every $t>0$,

(b) $(-1)^{d-2} \psi^{(d-2)}$ is decreasing and convex in $] 0,+\infty[$.

Example 6.1 (Gumbel-Hougaard copula). The standard expression for members of this family of $d$-copulas is

$$
C_{\theta}^{\mathbf{G H}}(\mathbf{u})=\exp \left(-\left(\sum_{i=1}^{d}\left(-\log \left(u_{i}\right)\right)^{\theta}\right)^{1 / \theta}\right)
$$

where $\theta \geq 1$. For $\theta=1$ we obtain the independence copula as a special case, and the limit of $C_{\theta}^{\mathbf{G H}}$ for $\theta \rightarrow+\infty$ is the comonotonicity copula. The Archimedean generator of this family is given by $\psi(t)=\exp \left(-t^{1 / \theta}\right)$. Each member of this class is absolutely continuous.

Copulas of this type can be derived from the work by Gumbel [90] and have been further considered by Hougaard [97]. For this reason, this family is named as Gumbel-Hougaard family [98].

Example 6.2 (Mardia-Takahasi-Clayton copula). The standard expression for members of this family of $d$-copulas is

$$
C_{\theta}^{\mathbf{M T C}}(u, v)=\max \left\{\left(\sum_{i=1}^{d} u_{i}^{-\theta}-(d-1)\right)^{-1 / \theta}, 0\right\}
$$

where $\theta \geq \frac{-1}{d-1}, \theta \neq 0$. The limiting case $\theta=0$ corresponds to the independence copula.

The Archimedean generator of this family is given by

$$
\psi_{\theta}(t)=(\max \{1+\theta t, 0\})^{-1 / \theta} .
$$

It was proved in [136] that, for every $d$-dimensional Archimedean copula $C$ and for every $\mathbf{u} \in \mathbb{I}^{d}, C_{\theta_{L}}^{\mathbf{M T C}}(\mathbf{u}) \leq C(\mathbf{u})$ for $\theta_{L}=-\frac{1}{d-1}$. 
Copulas of this type can be derived from two types of multivariate distributions: the Pareto distribution by Mardia [130] and the Burr distribution by Takahasi [194]. They were mentioned as bivariate copulas in [108] and as multivariate copulas in [21], subsequently extended in [80]. A special member of this family can also be derived from the bivariate logistic model of first type by Gumbel [91]. As showed by Oakes [157], bivariate copulas of type (19) are associated with the Clayton's model [20]. For this reason, many people referred to this family as the Clayton's family of copulas. Here, we prefer to name it as Mardia-Takahasi-Clayton family of copulas.

Example 6.3 (Frank copula). The standard expression for members of this family of $d$-copulas is

$$
C_{\theta}^{\mathbf{F r}}(\mathbf{u})=-\frac{1}{\theta} \log \left(1+\frac{\prod_{i=1}^{d}\left(\mathrm{e}^{-\theta u_{i}}-1\right)}{\left(\mathrm{e}^{-\theta}-1\right)^{d-1}}\right),
$$

where $\theta>0$. The limiting case $\theta=0$ corresponds to $\Pi_{d}$. For the case $d=2$, the parameter $\theta$ can be extended also to the case $\theta<0$.

Copulas of this type have been introduced by Frank [68] in relation with a problem about associative functions on $\mathbb{I}$. They are absolutely continuous.

The Archimedean generator is given by $\psi_{\theta}(t)=-\frac{1}{\theta} \log \left(1-\left(1-\mathrm{e}^{-\theta}\right) \mathrm{e}^{-t}\right)$.

\subsection{EFGM copulas}

The so-called Eyraud-Farlie-Gumbel-Morgenstern (shortly, EFGM) distributions have been considered by Morgenstern [145] and Gumbel [88, 89], further developed by Farlie [63]. However, the idea of considering such distributions originated in an earlier and, for many years, forgotten work by Eyraud [60]. Here, we present the EFGM family of copulas that can be derived from the papers just mentioned.

Let $d \geq 2$. Let $\mathcal{S}$ be the class of all subsets of $\{1,2, \ldots, d\}$ having at least 2 elements. Trivially, $\mathcal{S}$ contains $2^{d}-d-1$ elements. To each $S \in \mathcal{S}$, we associate a real number $\alpha_{S}$, with the convention that, when $S=\left\{i_{1}, i_{2}, \ldots, i_{k}\right\}, \alpha_{S}=\alpha_{i_{1} i_{2} \ldots i_{k}}$.

An EFGM copula can be expressed in the following form:

$$
C_{d}^{\mathbf{E F G M}}(\mathbf{u})=\prod_{i=1}^{d} u_{i}\left(1+\sum_{S \in \mathcal{S}} \alpha_{S} \prod_{j \in S}\left(1-u_{j}\right)\right),
$$

for suitable values of the $\alpha_{S}$ 's.

For the bivariate and trivariate cases, respectively, EFGM copulas have the following expressions:

$$
C_{2}^{\mathbf{E F G M}}\left(u_{1}, u_{2}\right)=u_{1} u_{2}\left(1+\alpha_{12}\left(1-u_{1}\right)\left(1-u_{2}\right)\right),
$$

and 


$$
\begin{aligned}
C_{3}^{\mathbf{E F G M}}\left(u_{1}, u_{2}, u_{3}\right)= & u_{1} u_{2} u_{3}\left[1+\alpha_{12}\left(1-u_{1}\right)\left(1-u_{2}\right)+\alpha_{13}\left(1-u_{1}\right)\left(1-u_{3}\right)\right. \\
& \left.+\alpha_{23}\left(1-u_{2}\right)\left(1-u_{3}\right)+\alpha_{123}\left(1-u_{1}\right)\left(1-u_{2}\right)\left(1-u_{3}\right)\right] .
\end{aligned}
$$

It is not so difficult to show that any FGM copula is absolutely continuous with density given by

$$
c_{d}^{\mathbf{E F G M}}(\mathbf{u})=1+\sum_{S \in \mathcal{S}} \alpha_{S} \prod_{j \in S}\left(1-2 u_{j}\right) .
$$

As a consequence, the parameters $\alpha_{S}$ 's have to satisfy the following inequality

$$
1+\sum_{S \in \mathcal{S}} \alpha_{S} \prod_{j \in S} \xi_{j} \geq 0
$$

for any $\xi_{j} \in\{-1,1\}$. In particular, it holds that $\left|\alpha_{S}\right| \leq 1$.

On account of the fact that EFGM copulas do not allow the modelling of large dependence among the random variables involved, several extensions have been proposed in the literature, starting with the works by Farlie [63] and Saramanov [177]. A complete survey about these generalized EFGM models of dependence is given in [40], where a list of several other references can be also found. More recent investigations are also provided in [4, 5, 6, 65, 169]. Another possible approach for extending EFGM copulas is based on the construction of copulas that are quadratic in one variable $[163,170]$.

\section{Costructions of copulas}

Several constructions of copulas have been developed during the years from a variety of perspectives. At an abstract level, all these methods start with some known copulas and/or some auxiliary functions (sometimes, possible sections of copulas) and generate in an automatic way "new" copulas. Essentially, three kinds of such constructions can be distinguished.

\subsection{Copulas with given lower dimensional marginals}

These constructions are strictly related to the original Fréchet problem of considering distribution functions with fixed marginals (eventually overlapping). In this context the most interesting results have been obtained by means of the "conditioning method", as used by Dall'Aglio [28, 29] and Rüschendorf [173]. Joe presented several results of this type in [103, chapter 3]. A powerful recent method based on these ideas is the so-called pair-copula construction (for more details, see [27] and the references therein). For related recent studies, see [46, 116]. Other constructions can be found in [162] (direct compatibility), [95] (nested constructions), and $[17,66,115,123]$. 


\subsection{Copula-to-copula transformations}

Constructions of this second kind aim at transforming $d$-copulas into other $d-$ copulas having possibly some additional features (for example, having a larger number of parameters). Specifically, the following cases have been extensively studied.

\subsubsection{Ordinal sums}

The ordinal sum construction for (bivariate) copulas is fully described in [150, 185]. This construction was introduced in an algebraic framework, namely the theory of semigroups. Then, it was translated into the language of triangular norms (briefly, $t$-norms), which are binary operations on II that are associative, commutative, monotonic and with neutral element 1 (see $[1,111,185]$ ), and, finally, it was applied as well to bivariate copulas (which, in fact, can be seen also as special binary operations on $\mathbb{I}$ ). An extension of the ordinal sum construction to $\mathcal{C}_{d}$ has been recently discussed in [138] and [101, 102]. This method is essentially based on a kind of "patchwork procedure", consisting of redefining the value that a copula assume on a $d$-box $B$ of $\mathbb{I}^{d}$ by plugging-in a suitable rescaling of another copula. For other recent investigations of this type, see [33, 50, 190]. Following [138], we give the following result.

Theorem 7.1. Let $\mathcal{J}$ be a finite or countable subset of $\mathbb{N}$ and let (]$a_{k}, b_{k}[)_{k \in \mathcal{J}}$ be a family of sub-intervals of $\mathbb{I}$ indexed by $\mathcal{J}$ and let $\left(C_{k}\right)_{k \in \mathcal{J}}$ be a family of copulas in $\mathcal{C}_{d}$ also indexed by $\mathcal{J}$. It is required that any two of the intervals $] a_{k}, b_{k}[(k \in \mathcal{J})$ have at most an endpoint in common. Then the ordinal sum $C$ of $\left(C_{k}\right)_{k \in \mathcal{J}}$ with respect to family of intervals (]$a_{k}, b_{k}[)_{k \in \mathcal{J}}$ is the $d$-copula defined, for all $\mathbf{u} \in \mathbb{I}^{d}$ by

$$
C(\mathbf{u}):=\left\{\begin{array}{c}
a_{k}+\left(b_{k}-a_{k}\right) C_{k}\left(\frac{\min \left\{u_{1}, b_{k}\right\}-a_{k}}{b_{k}-a_{k}}, \ldots, \frac{\min \left\{u_{d}, b_{k}\right\}-a_{k}}{b_{k}-a_{k}}\right), \\
\left.\quad \text { if } \min \left\{u_{1}, u_{2}, \ldots, u_{d}\right\} \in\right] a_{k}, b_{k}[\text { for some } k \in \mathcal{J}, \\
\min \left\{u_{1}, u_{2}, \ldots, u_{d}\right\}, \quad \text { elsewhere. }
\end{array}\right.
$$

For such a $C$ one writes $C=\left(\left\langle a_{k}, b_{k}, C_{k}\right\rangle\right)_{k \in \mathcal{J}}=\bigoplus_{\left\{\left(a_{k}, b_{k}\right): k \in \mathcal{J}\right\}} C_{k}$.

\subsubsection{Distortions}

Given a copula $C$ and an increasing bijection $\psi: \mathbb{I} \rightarrow \mathbb{I}$, the distortion of $C$ is defined as the function $C_{\psi}: \mathbb{I}^{d} \rightarrow \mathbb{I}$,

$$
C_{\psi}(\mathbf{u})=\psi\left(C\left(\psi^{-1}\left(u_{1}\right), \psi^{-1}\left(u_{2}\right), \ldots, \psi^{-1}\left(u_{d}\right)\right)\right) .
$$

Such a transformation has originated from the study of distorted probability distribution functions (especially, power distortions), and has been considered by several authors like $[3,18,42,52,54,73,85,112,113,150,146]$. In reliability the- 
ory, this kind of transformation is used in order to introduce the so-called bivariate ageing function that are used for the definition of bivariate notion of ageing (see $[8,9,53,148])$. In the context of synthetic Collateralized Debt Obligations (CDOs), distortions of copulas have been recently used in order to produce a heavy tailed portfolio loss distribution [22].

\subsubsection{Pointwise composition of copulas}

Given two copulas $A$ and $B$ in $\mathcal{C}_{d}$, we define as composition of $A$ and $B$ through some suitable functions $H: \mathbb{I}^{2} \rightarrow \mathbb{I}, f_{i}: \mathbb{I} \rightarrow \mathbb{I}$ and $g_{i}: \mathbb{I} \rightarrow \mathbb{I}(i=1,2, \ldots, d)$, any mapping $C_{A, B}: \mathbb{I}^{d} \rightarrow \mathbb{I}$ given by

$$
C_{A, B}(\mathbf{u})=H\left(A\left(f_{1}\left(u_{1}\right), \ldots, f_{d}\left(u_{d}\right)\right), B\left(g_{1}\left(u_{1}\right), \ldots, g_{d}\left(u_{d}\right)\right)\right) .
$$

To the best of our knowledgement, the idea of such constructions arose in the Khoudraji's work [107], and, then, studied and further generalized in [23, 41, 79, 124]. Although, formally, distortions of copulas can be included in this class (for a suitable choice of $H$ ), we prefer to distinguish these constructions, following their historical development.

\subsubsection{Shuffles of copulas}

These constructions are based on the transformation of a copula $C$ into another one by means of a suitable rearrangement of the original mass distribution of $C$. The idea goes back to the notion of shuffles of Min, as introduced in [143], and is related to some modifications of the copula $M_{d}$ (see also [141]). A recent generalization is discussed in [51].

\subsection{Geometric constructions of copulas}

The third kind of constructions refers to methods for originating copulas starting with some information about their structure (for example, support, diagonals, sections). For a good overview to these constructions, the reader should refer to [150, chapter 3]. More recent investigations are listed below.

- copulas with given support: see [72];

- copulas with given horizontal and/or vertical sections: see [48, 110, 167, 170, 196];

- copulas with given diagonal sections: see [34, 35, 44, 47, 59, 101, 102, 134, 154];

- copulas with given affine sections [109, 164]. 


\section{Copula theory: what's the future?}

Above we have described, sometimes in a sketchy manner, the state of some, if not most, of the known results about copula theory; thus, the title of the present section poses a very natural question. It is hard to foresee the future, but there certainly are a few directions that we feel the investigations about copulas and their applications are likely to take. Running the risk of being completely, or even partially, proved wrong, we venture to put forward the following suggestions for likely directions of future investigations:

New constructions of copulas. The search for families of copulas having properties desirable for specific applications in various fields ought to continue to be important. Having at one's disposal several families of copulas (spanning different behaviour) is essential in order to create a wider spectrum of possible scenarios for the stochastic model at hand. This is of special interest to assist in decision making the risk managers and, under Basel Accords, it is mandatory for large banks to determine their risky positions. In particular, we think that special emphasis will be devoted to the search for copulas exhibiting different asymmetries, (non-exchangeable copulas, copulas with different tail behaviour, etc.).

The compatibility problem. Given that one just has some vague idea about the dependence of a r.v. $\mathbf{X}$ (for example, one knows the lower dimensional marginals of $\mathbf{X}$ or some dependence measures among its components), the question is whether one can describe the set of all possible copulas of $\mathbf{X}$, compatible with the given information. As said, this problem has its roots at early works on the Fréchet classes, but its popularity have recently increased due to its connection with several problems arising in risk aggregation (see, e.g., [58]).

Copulas and stochastic processes. Starting with the seminal paper by Darsow, Nguyen and Olsen [32] linking copulas and Markov processes, it is still a matter of discussion whether copulas can be really useful for describing space-time dependence structure (see also [119]). In this respect, the recent concept of Lévycopula (see $[7,10,106,99]$ ) seems quite powerful and promising for modelling the large class of Lévy processes.

Other investigations related related to copulas and time-changing dependence structure can be found in [86, 159].

Acknowledgements First of all, we would like to express our gratitude to several Colleagues with whom through the years we had the pleasure to discuss ideas on copulas and their applications. Some of them have also received, and commented on, a first version of the present report. We thank them for their comments and suggestions: we feel that these have improved our presentation.

\section{References}

1. Alsina, C., Frank, M.J., Schweizer, B.: Associative functions. Triangular norms and copulas. World Scientific Publishing Co. Pte. Ltd., Hackensack, NJ (2006) 
2. Alsina, C., Nelsen, R.B., Schweizer, B.: On the characterization of a class of binary operations on distribution functions. Statist. Probab. Lett. 17 (2), 85-89 (1993)

3. Alvoni, E., Papini, P.L., Spizzichino, F.: On a class of transformations of copulas and quasicopulas. Fuzzy Sets and Systems 160 (3), 334-343 (2009)

4. Amblard, C., Girard, S.: Une famille semi-paramétrique de copules symétriques bivariées. C. R. Acad. Sci. Paris Sér. I Math. 333 (2), 129-132 (2001)

5. Amblard, C., Girard, S.: Symmetry and dependence properties within a semiparametric family of bivariate copulas. J. Nonparametr. Stat. 14 (6), 715-727 (2002)

6. Amblard, C., Girard, S.: A new symmetric extension of FGM copulas. Metrika 70 (1), 1-17 (2009)

7. Barndorff-Nielsen, O.E., Lindner, A.M.: Lévy copulas: dynamics and transforms of Upsilon type. Scand. J. Statist. 34 (2), 298-316 (2007)

8. Bassan, B., Spizzichino, F.: Bivariate survival models with Clayton aging functions. Insurance Math. Econom. 37 (1), 6-12 (2005)

9. Bassan, B., Spizzichino, F.: Relations among univariate aging, bivariate aging and dependence for exchangeable lifetimes. J. Multivariate Anal. 93 (2), 313-339 (2005)

10. Bäuerle, N., Blatter, A., Müller, A.: Dependence properties and comparison results for Lévy processes. Math. Methods Oper. Res. 67 (1), 161-186 (2008)

11. Beneš, V., Štěpán, J. (eds.): Distributions with given marginals and moment problems. Kluwer Academic Publishers, Dordrecht (1997)

12. Berg, D.: Copula goodness-of-fit testing. In: F. Durante, W. Härdle, P. Jaworski, T. Rychlik (eds.) Workshop on Copula Theory and its Applications. Springer, Dortrecht (NL) (2010)

13. Billingsley, P.: Probability and measure, third edn. Wiley Series in Probability and Mathematical Statistics. John Wiley \& Sons Inc., New York (1995). A Wiley-Interscience Publication

14. Burchard, A., Hajaiej, H.: Rearrangement inequalities for functionals with monotone integrands. J. Funct. Anal. 233 (2), 561-582 (2006)

15. Carley, H.: Maximum and minimum extensions of finite subcopulas. Comm. Statist. Theory Methods 31 (12), 2151-2166 (2002)

16. Carley, H., Taylor, M.D.: A new proof of Sklar's theorem. In: C.M. Cuadras, J. Fortiana, J.A. Rodriguez-Lallena (eds.) Distributions with given marginals and statistical modelling, pp. 29-34. Kluwer Acad. Publ., Dordrecht (2002)

17. Chakak, A., Koehler, K.J.: A strategy for constructing multivariate distributions. Comm. Statist. Simulation Comput. 24 (3), 537-550 (1995)

18. Charpentier, A.: Dynamic dependence ordering for Archimedean copulas and distorted copulas. Kybernetika (Prague) 44 (6), 777-794 (2008)

19. Cherubini, U., Luciano, E., Vecchiato, W.: Copula methods in finance. Wiley Finance Series. John Wiley \& Sons Ltd., Chichester (2004)

20. Clayton, D.G.: A model for association in bivariate life tables and its application in epidemiological studies of familial dependency in chronic disease incidence. Biometrika 65, 141-151 (1978)

21. Cook, R.D., Johnson, M.E.: A family of distributions for modelling nonelliptically symmetric multivariate data. J. Roy. Statist. Soc. Ser. B 43 (2), 210-218 (1981)

22. Crane, G., van der Hoek, J.: Using distortions of copulas to price synthetic CDOs. Insurance Math. Econom. 42 (3), 903-908 (2008)

23. Cuadras, C.M.: Constructing copula functions with weighted geometric means. J. Statist. Plan. Infer. 139 (11), 3766-3772 (2009)

24. Cuadras, C.M., Augé, J.: A continuous general multivariate distribution and its properties. Comm. Statist. A-Theory Methods 10 (4), 339-353 (1981)

25. Cuadras, C.M., Fortiana, J., Rodriguez-Lallena, J.A. (eds.): Distributions with given marginals and statistical modelling. Kluwer Academic Publishers, Dordrecht (2002). Papers from the meeting held in Barcelona, July 17-20, 2000

26. Cuculescu, I., Theodorescu, R.: Copulas: diagonals, tracks. Rev. Roumaine Math. Pures Appl. 46 (6), 731-742 (2002) (2001)

27. Czado, C.: Pair-copula constructions. In: F. Durante, W. Härdle, P. Jaworski, T. Rychlik (eds.) Workshop on Copula Theory and its Applications. Springer, Dortrecht (NL) (2010) 
28. Dall'Aglio, G.: Sulla compatibilità delle funzioni di ripartizione doppia. Rend. Mat. e Appl. (5) 18, 385-413 (1959)

29. Dall'Aglio, G.: Fréchet classes and compatibility of distribution functions. In: Symposia Mathematica, Vol IX (Convegno di Calcolo delle Probabilità, INDAM, Rome, 1971), pp. 131-150. Academic Press, London (1972)

30. Dall'Aglio, G.: Fréchet classes: the beginnings. In: G. Dall'Aglio, S. Kotz, G. Salinetti (eds.) Advances in probability distributions with given marginals (Rome, 1990), Math. Appl., vol. 67, pp. 1-12. Kluwer Acad. Publ., Dordrecht (1991)

31. Dall'Aglio, G., Kotz, S., Salinetti, G. (eds.): Advances in probability distributions with given marginals, Mathematics and its Applications, vol. 67. Kluwer Academic Publishers Group, Dordrecht (1991). Beyond the copulas, Papers from the Symposium on Distributions with Given Marginals held in Rome, April 1990

32. Darsow, W.F., Nguyen, B., Olsen, E.T.: Copulas and Markov processes. Illinois J. Math. 36 (4), 600-642 (1992)

33. De Baets, B., De Meyer, H.: Orthogonal grid constructions of copulas. IEEE Trans. Fuzzy Systems 15 (6), 1053-1062 (2007)

34. De Baets, B., De Meyer, H., Mesiar, R.: Asymmetric semilinear copulas. Kybernetika (Prague) 43 (2), 221-233 (2007)

35. De Baets, B., De Meyer, H., Úbeda-Flores, M.: Opposite diagonal sections of quasi-copulas and copulas. Internat. J. Uncertain. Fuzziness Knowledge-Based Systems 17 (4), 481-490 (2009)

36. Deheuvels, P.: Caractérisation complète des lois extrêmes multivariées et de la convergence des types extrêmes. Publ. Inst. Stat. Univ. Paris 23 (3-4), 1-36 (1978)

37. Deheuvels, P.: Indépendance multivariée partielle et inégalités de Fréchet. In: Studies in probability and related topics, pp. 145-155. Nagard, Rome (1983)

38. Dhaene, J., Denuit, M., Goovaerts, M.J., Kaas, R., Vyncke, D.: The concept of comonotonicity in actuarial science and finance: applications. Insurance Math. Econom. 31 (2), 133-161 (2002)

39. Dhaene, J., Denuit, M., Goovaerts, M.J., Kaas, R., Vyncke, D.: The concept of comonotonicity in actuarial science and finance: theory. Insurance Math. Econom. 31 (1), 3-33 (2002). 5th IME Conference (University Park, PA, 2001)

40. Drouet-Mari, D., Kotz, S.: Correlation and dependence. Imperial College Press, London (2001)

41. Durante, F.: Construction of non-exchangeable bivariate distribution functions. Statist. Papers 50 (2), 383-391 (2009)

42. Durante, F., Foschi, R., Sarkoci, P.: Distorted copulas: constructions and tail dependence. Comm. Statist. Theory Methods (2010). In press

43. Durante, F., Hofert, M., Scherer, M.: Multivariate hierarchical copulas with shocks. Methodol. Comput. Appl. Probab. (2009). In press

44. Durante, F., Jaworski, P.: Absolutely continuous copulas with given diagonal sections. Comm. Statist. Theory Methods 37 (18), 2924-2942 (2008)

45. Durante, F., Klement, E., Quesada-Molina, J.: Bounds for trivariate copulas with given bivariate marginals. J. Inequal. Appl. 2008, 1-9 (2008). Article ID 161537

46. Durante, F., Klement, E., Quesada-Molina, J., Sarkoci, P.: Remarks on two product-like constructions for copulas. Kybernetika (Prague) 43 (2), 235-244 (2007)

47. Durante, F., Kolesárová, A., Mesiar, R., Sempi, C.: Copulas with given diagonal sections: novel constructions and applications. Internat. J. Uncertain. Fuzziness Knowledge-Based Systems 15 (4), 397-410 (2007)

48. Durante, F., Kolesárová, A., Mesiar, R., Sempi, C.: Copulas with given values on a horizontal and a vertical section. Kybernetika (Prague) 43 (2), 209-220 (2007)

49. Durante, F., Quesada-Molina, J.J., Úbeda-Flores, M.: On a family of multivariate copulas for aggregation processes. Inform. Sci. 177 (24), 5715-5724 (2007)

50. Durante, F., Saminger-Platz, S., Sarkoci, P.: Rectangular patchwork for bivariate copulas and tail dependence. Comm. Statist. Theory Methods 38 (15), 2515-2527 (2009) 
51. Durante, F., Sarkoci, P., Sempi, C.: Shuffles of copulas. J. Math. Anal. Appl. 352 (2), 914 921 (2009)

52. Durante, F., Sempi, C.: Copula and semicopula transforms. Int. J. Math. Math. Sci. 2005 (4), 645-655 (2005)

53. Durante, F., Spizzichino, F.: Semi-copulas, capacities and families of level curves. Fuzzy Sets and Systems (2009). In press

54. Durrleman, V., Nikeghbali, A., Roncalli, T.: A simple transformation of copulas (2000). Available at SSRN: http://ssrn.com/abstract=1032543

55. Embrechts, P.: Discussion of: "Copulas: tales and facts" by T. Mikosch [Extremes 9 (2006), no. 1, 3-20]. Extremes 9 (1), 45-47 (2006)

56. Embrechts, P.: Copulas: a personal view. J. Risk Ins. 76 (3), 639-650 (2009)

57. Embrechts, P., McNeil, A.J., Straumann, D.: Correlation and dependence in risk management: properties and pitfalls. In: M. Dempster (ed.) Risk management: value at risk and beyond, pp. 176-223. Cambridge University Press, Cambridge (2002)

58. Embrechts, P., Puccetti, G.: Risk aggregation. In: F. Durante, W. Härdle, P. Jaworski, T. Rychlik (eds.) Workshop on Copula Theory and its Applications. Springer, Dortrecht (NL) (2010)

59. Erdely, A., González-Barrios, J.M.: On the construction of families of absolutely continuous copulas with given restrictions. Comm. Statist. Theory Methods 35 (4-6), 649-659 (2006)

60. Eyraud, H.: Les principes de la mesure des correlations. Ann. Univ. Lyon, III. Ser., Sect. A 1, 30-47 (1936)

61. Fang, H.B., Fang, K.T., Kotz, S.: The meta-elliptical distributions with given marginals. J. Multivariate Anal. 82 (1), 1-16 (2002)

62. Fang, K.T., Kotz, S., Ng, K.W.: Symmetric multivariate and related distributions, Monographs on Statistics and Applied Probability, vol. 36. Chapman and Hall Ltd., London (1990)

63. Farlie, D.J.G.: The performance of some correlation coefficients for a general bivariate distribution. Biometrika 47, 307-323 (1960)

64. Féron, R.: Sur les tableaux de corrélation dont les marges sont données. Cas de l'espace a trois dimensions. Publ. Inst. Statist. Univ. Paris 5, 3-12 (1956)

65. Fischer, M., Klein, I.: Constructing generalized FGM copulas by means of certain univariate distributions. Metrika 65 (2), 243-260 (2007)

66. Fischer, M., Köck, C., Schlüter, S., Weigert, F.: An empirical analysis of multivariate copula models. Quant. Finance (2009). In press

67. Frahm, G., Junker, M., Szimayer, A.: Elliptical copulas: applicability and limitations. Statist. Probab. Lett. 63 (3), 275-286 (2003)

68. Frank, M.J.: On the simultaneous associativity of $F(x, y)$ and $x+y-F(x, y)$. Aequationes Math. 19 (2-3), 194-226 (1979)

69. Fréchet, M.: Sur les tableaux de corrélation dont les marges sont données. Ann. Univ. Lyon. Sect. A. (3) 14, 53-77 (1951)

70. Fréchet, M.: Sur les tableaux de corrélation dont les marges sont données. C. R. Acad. Sci. Paris 242, 2426-2428 (1956)

71. Fréchet, M.: Remarques au sujet de la note précédente. C. R. Acad. Sci. Paris 246, 27192720 (1958)

72. Fredricks, G.A., Nelsen, R.B., Rodríguez-Lallena, J.A.: Copulas with fractal supports. Insurance Math. Econom. 37 (1), 42-48 (2005)

73. Frees, E.W., Valdez, E.A.: Understanding relationships using copulas. N. Am. Actuar. J. 2 (1), 1-25 (1998)

74. Genest, C.: Preface [International Conference on Dependence Modelling: Statistical Theory and Applications in Finance and Insurance (DeMoSTAFI)]. Canad. J. Statist. 33 (3), 313-314 (2005). Held in Québec City, QC, May 20-22, 2004

75. Genest, C.: Preface [Special issue: Papers presented at the DeMoSTAFI Conference]. Insurance Math. Econom. 37 (1), 1-2 (2005). Held in Québec, QC, May 20-22, 2004

76. Genest, C., Favre, A.C.: Everything you always wanted to know about copula modeling but were afraid to ask. J. Hydrol. Eng. 12 (4), 347-368 (2007) 
77. Genest, C., Favre, A.C., Béliveau, J., Jacques, C.: Metaelliptical copulas and their use in frequency analysis of multivariate hydrological data. Water Resour. Res. 43, W09,401 (2007). Doi:10.1029/2006WR005275

78. Genest, C., Gendron, M., Bourdeau-Brien, M.: The advent of copulas in finance. Europ. J. Finance 53 (15), in press (2009)

79. Genest, C., Ghoudi, K., Rivest, L.P.: "Understanding relationships using copulas," by Edward Frees and Emiliano Valdez, January 1998. N. Am. Actuar. J. 2 (3), 143-149 (1998)

80. Genest, C., MacKay, R.J.: Copules archimédiennes et familles de lois bidimensionnelles dont les marges sont données. Canad. J. Statist. 14 (2), 145-159 (1986)

81. Genest, C., MacKay, R.J.: The joy of copulas: bivariate distributions with uniform marginals. Amer. Statist. 40 (4), 280-283 (1986)

82. Genest, C., Nešlehová, J.: A primer on copulas for count data. Astin Bull. 37 (2), 475-515 (2007)

83. Genest, C., Quesada-Molina, J.J., Rodríguez-Lallena, J.A., Sempi, C.: A characterization of quasi-copulas. J. Multivariate Anal. 69 (2), 193-205 (1999)

84. Genest, C., Rémillard, B.: Discussion of: "Copulas: tales and facts" by T. Mikosch [Extremes 9 (2006), no. 1, 3-20]. Extremes 9 (1), 27-36 (2006)

85. Genest, C., Rivest, L.P.: On the multivariate probability integral transformation. Statist. Probab. Lett. 53 (4), 391-399 (2001)

86. Giacomini, E., Härdle, W., Spokoiny, V.: Inhomogeneous dependence modeling with timevarying copulae. J. Bus. Econom. Statist. 27 (2), 224-234 (2009)

87. Gudendorf, G., Segers, J.: Extreme value theory and copulae. In: F. Durante, W. Härdle, P. Jaworski, T. Rychlik (eds.) Workshop on Copula Theory and its Applications. Springer, Dortrecht (NL) (2010)

88. Gumbel, E.J.: Distributions à plusieurs variables dont les marges sont données. C. R. Acad. Sci. Paris 246, 2717-2719 (1958)

89. Gumbel, E.J.: Bivariate exponential distributions. J. Amer. Statist. Assoc. 55, 698-707 (1960)

90. Gumbel, E.J.: Distributions des valeurs extrêmes en plusieurs dimensions. Publ. Inst. Statist. Univ. Paris 9, 171-173 (1960)

91. Gumbel, E.J.: Bivariate logistic distributions. J. Amer. Statist. Assoc. 56, 335-349 (1961)

92. de Haan, L.: Discussion of: "Copulas: tales and facts" by T. Mikosch [Extremes 9 (2006), no. 1, 3-20]. Extremes 9 (1), 21-22 (2006)

93. Härdle, W., Okhrin, O.: De copulis non est disputandum. Copulae: an overview. AStA Adv. Stat. Anal. (2009). In press

94. Hoeffding, W.: Maßstabinvariante Korrelationstheorie. Schriften des Mathematischen Instituts und des Instituts für Angewandte Mathematik der Universität Berlin 5 (3), 179-233 (1940). (Reprinted as "Scale-invariant correlation theory" in Fisher, N. I. and Sen, P. K., editors, The Collected Works of Wassily Hoeffding, pages 57-107. Springer, New York. 1994)

95. Hofert, M.: Construction and sampling of nested Archimedean copulas. In: F. Durante, W. Härdle, P. Jaworski, T. Rychlik (eds.) Workshop on Copula Theory and its Applications. Springer, Dortrecht (NL) (2010)

96. Höffding, W.: Maszstabinvariante Korrelationstheorie. Schr. Math. Inst. u. Inst. Angew. Math. Univ. Berlin 5, 181-233 (1940)

97. Hougaard, P.: A class of multivariate failure time distributions. Biometrika 73 (3), 671-678 (1986)

98. Hutchinson, T.P., Lai, C.D.: Continuous bivariate distributions, emphasising applications. Rumsby Scientific Publishing, Adelaide (1990)

99. Ibragimov, R.: Copula-based characterizations for higher order Markov processes. Economet. Theor. 25 (3), 819-846 (2009)

100. Ibragimov, R., Permiakova, E.: Copula estimation. In: F. Durante, W. Härdle, P. Jaworski, T. Rychlik (eds.) Workshop on Copula Theory and its Applications. Springer, Dortrecht (NL) (2010)

101. Jaworski, P.: On copulas and their diagonals. Inform. Sci. 179 (17), 2863-2871 (2009) 
102. Jaworski, P., Rychlik, T.: On distributions of order statistics for absolutely continuous copulas with applications to reliability. Kybernetika (Prague) 44 (6), 757-776 (2008)

103. Joe, H.: Multivariate models and dependence concepts, Monographs on Statistics and Applied Probability, vol. 73. Chapman \& Hall, London (1997)

104. Joe, H.: Discussion of: "Copulas: tales and facts" by T. Mikosch [Extremes 9 (2006), no. 1, 3-20]. Extremes 9 (1), 37-41 (2006)

105. Kallenberg, O.: Foundations of modern probability, second edn. Probability and its Applications (New York). Springer-Verlag, New York (2002)

106. Kallsen, J., Tankov, P.: Characterization of dependence of multidimensional Lévy processes using Lévy copulas. J. Multivariate Anal. 97 (7), 1551-1572 (2006)

107. Khoudraji, A.: Contributions à l'étude des copules et à la modélisation des valeurs extrêmes bivariées. Ph.D. thesis, Université de Laval, Québec (Canada) (1995)

108. Kimeldorf, G., Sampson, A.: Uniform representations of bivariate distributions. Comm. Statist. 4 (7), 617-627 (1975)

109. Klement, E.P., Kolesárová, A.: Intervals of 1-Lipschitz aggregation operators, quasi-copulas, and copulas with given affine section. Monatsh. Math. 152 (2), 151-167 (2007)

110. Klement, E.P., Kolesárová, A., Mesiar, R., Sempi, C.: Copulas constructed from horizontal sections. Comm. Statist. Theory Methods 36 (13-16), 2901-2911 (2007)

111. Klement, E.P., Mesiar, R., Pap, E.: Triangular norms, Trends in Logic-Studia Logica Library, vol. 8. Kluwer Academic Publishers, Dordrecht (2000)

112. Klement, E.P., Mesiar, R., Pap, E.: Archimax copulas and invariance under transformations. C. R. Math. Acad. Sci. Paris 340 (10), 755-758 (2005)

113. Klement, E.P., Mesiar, R., Pap, E.: Transformations of copulas. Kybernetika (Prague) 41 (4), 425-434 (2005)

114. Klüppelberg, C., Resnick, S.I.: The Pareto copula, aggregation of risks, and the emperor's socks. J. Appl. Probab. 45 (1), 67-84 (2008)

115. Koehler, K.J., Symanowski, J.T.: Constructing multivariate distributions with specific marginal distributions. J. Multivariate Anal. 55 (2), 261-282 (1995)

116. Kolesárová, A., Mesiar, R., Sempi, C.: Measure-preserving transformations, copulæ and compatibility. Mediterr. J. Math. 5 (3), 325-339 (2008)

117. Kolev, N., dos Anjos, U., Mendes, B.: Copulas: a review and recent developments. Stoch. Models 22 (4), 617-660 (2006)

118. Kollo, T.: Preface. J. Statist. Plan. Infer. 139 (11), 3740 (2009)

119. Lagerås, A.N.: Copulas for Markovian dependence. Bernoulli (2009). In press

120. Li, D.: On default correlation: a copula function approach. Journal of Fixed Income 9, 43-54 (2001)

121. Li, H.: Duality of he multivariate distributions of Marshall-Olkin type and tail dependence. Comm. Statist. Theory Methods 37 (11-12), 1721-1733 (2008)

122. Li, H.: Tail dependence comparison of survival Marshall-Olkin copulas. Methodol. Comput. Appl. Probab. 10 (1), 39-54 (2008)

123. Li, H., Scarsini, M., Shaked, M.: Linkages: a tool for the construction of multivariate distributions with given nonoverlapping multivariate marginals. J. Multivariate Anal. 56 (1), 20-41 (1996)

124. Liebscher, E.: Construction of asymmetric multivariate copulas. J. Multivariate Anal. 99 (10), 2234-2250 (2008)

125. Lindner, A.: Discussion of: "Copulas: tales and facts" by T. Mikosch [Extremes 9 (2006), no. 1, 3-20]. Extremes 9 (1), 43-44 (2006)

126. Mai, J.F., Scherer, M.: Efficiently sampling exchangeable Cuadras-Augé copulas in high dimensions. Inform. Sci. 179 (17), 2872-2877 (2009)

127. Mai, J.F., Scherer, M.: Lévy-Frailty copulas. J. Multivariate Anal. 100 (7), 1567-1585 (2009)

128. Mai, J.F., Scherer, M.: Reparameterizing Marshall-Olkin copulas with applications to highdimensional sampling. J. Stat. Comput. Simul. (2009) In press

129. Malevergne, Y., Sornette, D.: Extreme financial risks. Springer-Verlag, Berlin (2006)

130. Mardia, K.V.: Multivariate Pareto distributions. Ann. Math. Statist. 33, 1008-1015 (1962) 
131. Mardia, K.V.: Families of bivariate distributions. Hafner Publishing Co., Darien, Conn. (1970). Griffin's Statistical Monographs and Courses, No. 27

132. Marshall, A.W.: Copulas, marginals, and joint distributions. In: Distributions with fixed marginals and related topics (Seattle, WA, 1993), IMS Lecture Notes Monogr. Ser., vol. 28, pp. 213-222. Inst. Math. Statist., Hayward, CA (1996)

133. Marshall, A.W., Olkin, I.: A multivariate exponential distribution. J. Amer. Statist. Assoc. 62, 30-44 (1967)

134. Mayor, G., Mesiar, R., Torrens, J.: On quasi-homogeneous copulas. Kybernetika (Prague) 44 (6), 745-756 (2008)

135. Mc Neil, A.J., Nešlehová, J.: From Archimedean to Liouville copulas (2009). Submitted

136. Mc Neil, A.J., Nešlehová, J.: Multivariate Archimedean copulas, d-monotone functions and $\ell_{1}$-norm symmetric distributions. Ann. Statist. 37 (5B), 3059-3097 (2009)

137. McNeil, A.J., Frey, R., Embrechts, P.: Quantitative risk management. Concepts, techniques and tools. Princeton Series in Finance. Princeton University Press, Princeton, NJ (2005)

138. Mesiar, R., Sempi, C.: Ordinal sums and idempotents of copulas. Aequationes Math. (2009). In press

139. Mikosch, T.: Copulas: tales and facts. Extremes 9 (1), 3-20 (2006)

140. Mikosch, T.: "Copulas: tales and facts" [Extremes 9 (2006), no. 1, 3-20]-rejoinder. Extremes 9 (1), 55-62 (2006)

141. Mikusiński, P., , Taylor, M.D.: Some approximations of $n$-copulas. Metrika (2009). In press

142. Mikusiński, P., Sherwood, H., Taylor, M.D.: Probabilistic interpretations of copulas and their convex sums. In: Advances in probability distributions with given marginals (Rome, 1990), Math. Appl., vol. 67, pp. 95-112. Kluwer Acad. Publ., Dordrecht (1991)

143. Mikusiński, P., Sherwood, H., Taylor, M.D.: Shuffles of Min. Stochastica 13 (1), 61-74 (1992)

144. Moore, D.S., Spruill, M.C.: Unified large-sample theory of general chi-squared statistics for tests of fit. Ann. Statist. 3, 599-616 (1975)

145. Morgenstern, D.: Einfache Beispiele zweidimensionaler Verteilungen. Mitteilungsbl. Math. Statist. 8, 234-235 (1956)

146. Morillas, P.M.: A method to obtain new copulas from a given one. Metrika 61 (2), 169-184 (2005)

147. Nadarajah, S.: Marshall and Olkin's distributions. Acta Appl. Math. 103 (1), 87-100 (2008)

148. Nappo, G., Spizzichino, F.: Kendall distributions and level sets in bivariate exchangeable survival models. Inform. Sci. 179 (17), 2878-2890 (2009)

149. Nelsen, R.B.: An introduction to copulas, Lecture Notes in Statistics, vol. 139. SpringerVerlag, New York (1999)

150. Nelsen, R.B.: An introduction to copulas, second edn. Springer Series in Statistics. Springer, New York (2006)

151. Nelsen, R.B., Quesada-Molina, J.J., Rodríguez-Lallena, J.A., Úbeda-Flores, M.: Bounds on bivariate distribution functions with given margins and measures of association. Comm. Statist. Theory Methods 30 (6), 1155-1162 (2001)

152. Nelsen, R.B., Quesada-Molina, J.J., Rodríguez-Lallena, J.A., Úbeda-Flores, M.: Some new properties of quasi-copulas. In: C. Cuadras, J. Fortiana, J. Rodrí guez Lallena (eds.) Distributions with given marginals and Statistical Modelling, pp. 187-194. Kluwer, Dordrecht (2003)

153. Nelsen, R.B., Quesada-Molina, J.J., Rodríguez-Lallena, J.A., Úbeda-Flores, M.: Bestpossible bounds on sets of bivariate distribution functions. J. Multivariate Anal. 90 (2), 348-358 (2004)

154. Nelsen, R.B., Quesada-Molina, J.J., Rodríguez-Lallena, J.A., Úbeda-Flores, M.: On the construction of copulas and quasi-copulas with given diagonal sections. Insurance Math. Econom. 42 (2), 473-483 (2008)

155. Nelsen, R.B., Úbeda-Flores, M.: A comparison of bounds on sets of joint distribution functions derived from various measures of association. Comm. Statist. Theory Methods 33 (10), 2299-2305 (2004) 
156. Nelsen, R.B., Úbeda-Flores, M.: The lattice-theoretic structure of sets of bivariate copulas and quasi-copulas. C. R. Math. Acad. Sci. Paris 341 (9), 583-586 (2005)

157. Oakes, D.: A model for association in bivariate survival data. J. Roy. Statist. Soc. Ser. B 44 (3), 414-422 (1982)

158. Owzar, K., Sen, P.K.: Copulas: concepts and novel applications. Metron 61 (3), 323-353 (2004) (2003)

159. Patton, A.J.: Copula-based models for financial time series. In: T.G. Andersen, R.A. Davis, J.P. Kreiss, T. Mikosch (eds.) Handbook of Financial Time Series, pp. 767-785. Springer (2009)

160. Peng, L.: Discussion of: "Copulas: tales and facts" by T. Mikosch [Extremes 9 (2006), no. 1, 3-20]. Extremes 9 (1), 49-50 (2006)

161. Puccetti, G., Scarsini, M.: Multivariate comonotonicity. J. Multivariate Anal. (2009). In press

162. Quesada-Molina, J.J., Rodríguez-Lallena, J.A.: Some advances in the study of the compatibility of three bivariate copulas. J. Ital. Stat. Soc. 3 (3), 397-417 (1994)

163. Quesada-Molina, J.J., Rodríguez-Lallena, J.A.: Bivariate copulas with quadratic sections. J. Nonparametr. Statist. 5 (4), 323-337 (1995)

164. Quesada-Molina, J.J., Saminger-Platz, S., Sempi, C.: Quasi-copulas with a given subdiagonal section. Nonlinear Anal. 69 (12), 4654-4673 (2008)

165. Rényi, A.: On measures of dependence. Acta Math. Acad. Sci. Hungar. 10, 441-451 (1959)

166. Resnick, S.I.: Extreme values, regular variation, and point processes, Applied Probability. A Series of the Applied Probability Trust, vol. 4. Springer-Verlag, New York (1987)

167. Rodríguez-Lallena, J.A.: A class of copulas with piecewise linear horizontal sections. J. Statist. Plan. Infer. 139 (11), 3908-3920 (2009)

168. Rodríguez-Lallena, J.A., Úbeda-Flores, M.: Best-possible bounds on sets of multivariate distribution functions. Comm. Statist. Theory Methods 33 (4), 805-820 (2004)

169. Rodríguez-Lallena, J.A., Úbeda-Flores, M.: A new class of bivariate copulas. Statist. Probab. Lett. 66 (3), 315-325 (2004)

170. Rodríguez-Lallena, J.A., Úbeda-Flores, M.: Multivariate copulas with quadratic sections in one variable. Metrika (2009). In press

171. Rodríguez-Lallena, J.A., Úbeda-Flores, M.: Some new characterizations and properties of quasi-copulas. Fuzzy Sets and Systems 160 (6), 717-725 (2009)

172. Rüschendorf, L.: Sharpness of Fréchet-bounds. Z. Wahrsch. Verw. Gebiete 57 (2), 293-302 (1981)

173. Rüschendorf, L.: Construction of multivariate distributions with given marginals. Ann. Inst. Statist. Math. 37 (2), 225-233 (1985)

174. Rüschendorf, L.: On the distributional transform, Sklar's Theorem, and the empirical copula process. J. Statist. Plan. Infer. 139 (11), 3921-3927 (2009)

175. Rüschendorf, L., Schweizer, B., Taylor, M. (eds.): Distributions with fixed marginals and related topics, Institute of Mathematical Statistics Lecture Notes-Monograph Series, 28. Institute of Mathematical Statistics, Hayward, CA (1996)

176. Salvadori, G., De Michele, C., Kottegoda, N.T., Rosso, R.: Extremes in Nature. An Approach Using Copulas, Water Science and Technology Library, vol. 56. Springer, Dordrecht (NL) (2007)

177. Sarmanov, O.V.: Generalized normal correlation and two-dimensional Fréchet classes. Dokl. Akad. Nauk SSSR 168, 32-35 (1966)

178. Scarsini, M.: Copulae of probability measures on product spaces. J. Multivariate Anal. 31 (2), 201-219 (1989)

179. Schmid, F.: Copula-based measures of multivariate association. In: F. Durante, W. Härdle, P. Jaworski, T. Rychlik (eds.) Workshop on Copula Theory and its Applications. Springer, Dortrecht (NL) (2010)

180. Schönbucker, P.: Credit Derivatives Pricing Models: Models, Pricing, Implementation. Wiley Finance Series. John Wiley \& Sons Ltd., Chichester (2003)

181. Schweizer, B.: Thirty years of copulas. In: G. Dall'Aglio, S. Kotz, G. Salinetti (eds.) Advances in probability distributions with given marginals (Rome, 1990), Math. Appl., vol. 67, pp. 13-50. Kluwer Acad. Publ., Dordrecht (1991) 
182. Schweizer, B.: Introduction to copulas. J. Hydrol. Eng. 12 (4), 346-346 (2007)

183. Schweizer, B., Sklar, A.: Espaces métriques aléatoires. C. R. Acad. Sci. Paris 247, 20922094 (1958)

184. Schweizer, B., Sklar, A.: Operations on distribution functions not derivable from operations on random variables. Studia Math. 52, 43-52 (1974)

185. Schweizer, B., Sklar, A.: Probabilistic metric spaces. North-Holland Series in Probability and Applied Mathematics. North-Holland Publishing Co., New York (1983)

186. Schweizer, B., Wolff, E.F.: Sur une mesure de dépendance pour les variables aléatoires. C. R. Acad. Sci. Paris Sér. A 283, 659-661 (1976)

187. Schweizer, B., Wolff, E.F.: On nonparametric measures of dependence for random variables. Ann. Statist. 9 (4), 879-885 (1981)

188. Segers, J.: Efficient estimation of copula parameter. Discussion of: "Copulas: tales and facts" [Extremes 9 (2006), no. 1, 3-20] by T. Mikosch. Extremes 9 (1), 51-53 (2006)

189. Sempi, C.: Copulæ and their uses. In: K. Doksum, B. Lindquist (eds.) Mathematical and Statistical Methods in Reliability, pp. 73-86. World Scientific, Singapore (2003)

190. Siburg, K.F., Stoimenov, P.A.: Gluing copulas. Comm. Statist. Theory Methods 37 (19), 3124-3134 (2008)

191. Sklar, A.: Fonctions de répartition à $n$ dimensions et leurs marges. Publ. Inst. Statist. Univ. Paris 8, 229-231 (1959)

192. Sklar, A.: Random variables, joint distribution functions, and copulas. Kybernetika (Prague) 9, 449-460 (1973)

193. Sklar, A.: Random variables, distribution functions, and copulas-a personal look backward and forward. In: Distributions with fixed marginals and related topics (Seattle, WA, 1993), IMS Lecture Notes Monogr. Ser., vol. 28, pp. 1-14. Inst. Math. Statist., Hayward, CA (1996)

194. Takahasi, K.: Note on the multivariate Burr's distribution. Ann. Inst. Statist. Math. 17, 257$260(1965)$

195. Trivedi, P.K., Zimmer, D.M.: Copula Modeling: An Introduction for Practitioners, vol. 1 (2005)

196. Úbeda-Flores, M.: Multivariate copulas with cubic sections in one variable. J. Nonparametr. Statist. 20 (1), 91-98 (2008)

197. Whitehouse, M.: How a formula ignited market that burned some big investors. The Wall Street Journal (2005). Published on September 12, 2005

198. Williams, D.: Probability with martingales. Cambridge Mathematical Textbooks. Cambridge University Press, Cambridge (1991)

199. Wolff, E.F.: Measures of dependence derived from copulas. Ph.D. thesis, University of Massachusetts, Amherst (1977)

200. Wolff, E.F.: $n$-dimensional measures of dependence. Stochastica 4 (3), 175-188 (1980) 